\title{
Muséologies
}

Les cahiers d'études supérieures

muséologies

\section{Norman Slater — Leçons de design}

\section{Alessandra Mariani}

Volume 6, numéro 1, 2012

URI : https://id.erudit.org/iderudit/1011537ar

DOI : https://doi.org/10.7202/1011537ar

Aller au sommaire du numéro

Éditeur(s)

Association Québécoise de Promotion des Recherches Étudiantes en Muséologie (AQPREM)

ISSN

1718-5181 (imprimé)

1929-7815 (numérique)

Découvrir la revue

Citer ce compte rendu

Mariani, A. (2012). Compte rendu de [Norman Slater — Leçons de design]. Muséologies, 6(1), 151-157. https://doi.org/10.7202/1011537ar

Tous droits réservés (C Association Québécoise de Promotion des Recherches Étudiantes en Muséologie (AQPREM), 2012
Ce document est protégé par la loi sur le droit d'auteur. L’utilisation des services d'Érudit (y compris la reproduction) est assujettie à sa politique d'utilisation que vous pouvez consulter en ligne.

https://apropos.erudit.org/fr/usagers/politique-dutilisation/ 
Carnet

\section{Norman Slater | Leçons de design}

Alessandra Mariani

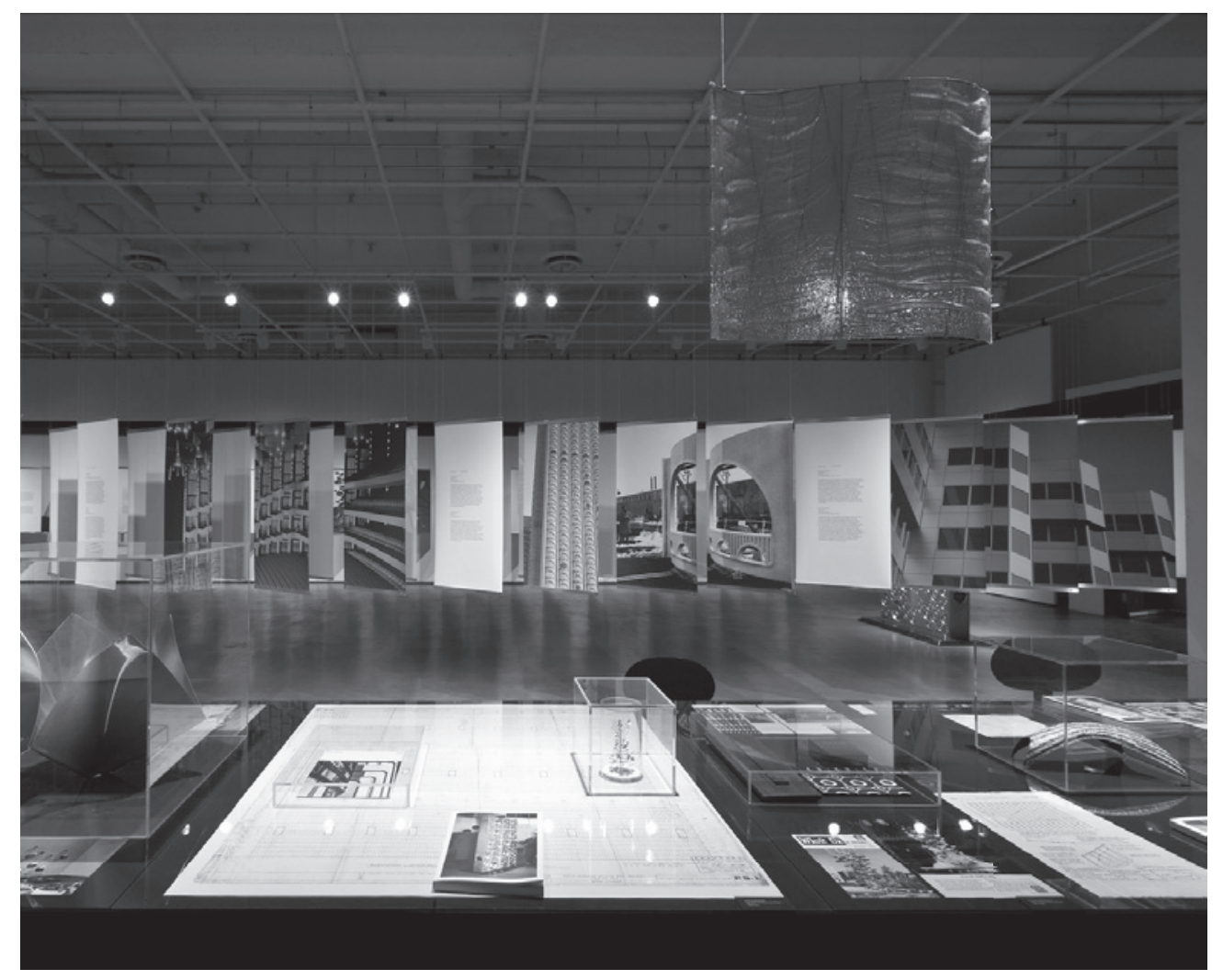

Vue de l'exposition

Photo: Michel Brunelle 

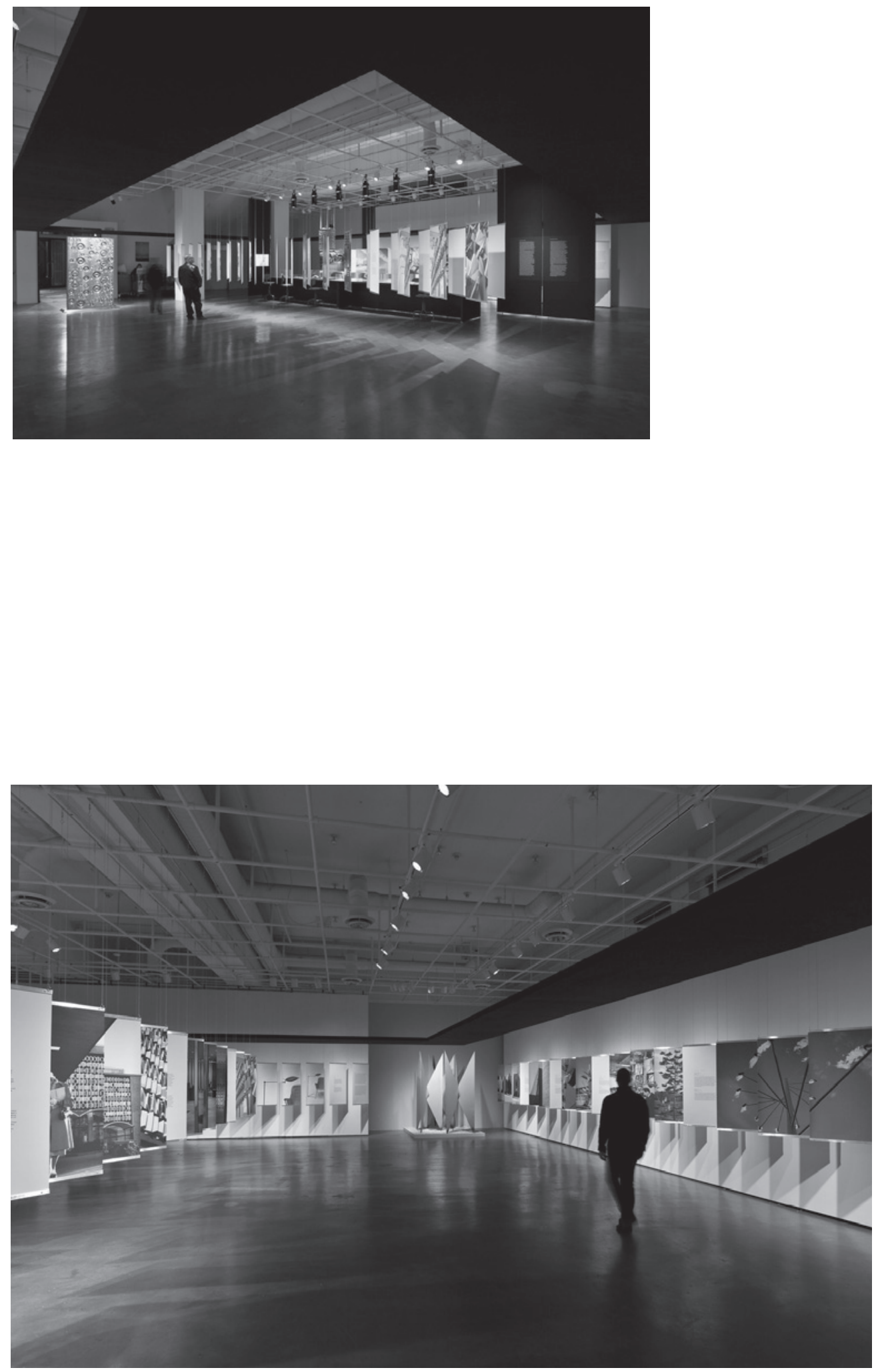

Vues de l'exposition

Photos: Michel Brunelle 
Le Centre de design de l'Université du Québec à Montréal (UQAM) a présenté du 24 novembre 2011 au 29 janvier 2012 l'exposition Norman Slater/Leçons de design sous le commissariat de Réjean Legault, professeur à l'École de design de I'UQAM. Le travail de Norman Slater, architecte, designer et artiste peu connu, a toute son importance pour la ville de Montréal puisqu'il s'est trouvé, à divers moments, intégré à plusieurs symboles architecturaux et urbains de la ville, dont: la Place des Arts (la grille ornementale de la salle Wilfrid-Pelletier, le dispositif d'éclairage de l'esplanade), le château Champlain (le mur-rideau caractéristique en béton préfabriqué), le Métro (l'uniformisation visuelle du réseau et la conception des équipements standards) et le parc d'attraction La Ronde, situé sur l'île Sainte-Hélène (le système d'éclairage du site). Si certaines de ses réalisations ont aujourd'hui disparu, l'exposition, scandée par 25 projets réalisés entre 1957 et 1983, aura réanimé momentanément l'univers créatif de Slater tout en révélant la diversité de son œuvre.

Le cursus d'études de Norman Slater, Montréalais d'origine, est significatif: après avoir obtenu son diplôme d'architecture à l'Université McGill (aux côtés d'un étudiant plus notoire, Arthur Erickson), il prolongea ses études à l'Institute of Design de Chicago puis au Royal College of Arts à Londres. Cette dernière phase de formation orienta véritablement sa carrière, puisqu'à son retour à Montréal c'est dans cet esprit qu'il produira la plus grande partie de son travail. Ce travail dérogeant des normes, polymorphe, est dû à sa volonté d'aller chercher plus loin que la pratique habituelle du design, afin de combler les lacunes des zones interstitielles qui se trouvent à la frontière de l'architecture, de l'art et de l'urbanisme.

Norman Slater (1921-2003) s'est révélé être, dans cette exposition, une des figures emblématiques de la fébrilité créative qui prévalait au cours des années 1950-1960 au Québec. Présenté comme un artiste de l'art appliqué, on comprend qu'il s'est rapidement frayé un chemin dans les sphères nationale (Slater a réalisé plusieurs œuvres et éléments décoratifs à Québec, Ottawa, Edmonton et Vancouver) et internationale, notamment par sa participation au pavillon canadien de l'exposition universelle de 1958 en Belgique (dont le lingot sculpté exposé dans le sas d'entrée du Centre de design), et par la fabrication d'une grille ornementale pour une filiale japonaise de l'Alcan à Tokyo en 1963. De toutes les matières qu'il a eu à façonner, l'aluminium semble être celle qui lui aura insufflé le plus d'énergie créatrice, et ce, dès le début de sa carrière. Selon Réjean Legault, «l'intérêt de Slater pour l'aluminium était en symbiose avec les stratégies de promotion du matériau soutenues par l'industrie $»^{1}$ : en fait, la malléabilité de ce matériau moderne était synchrone au développement de la pratique de Slater, plutôt expérimentale et sans considération pour les carcans disciplinaires. 

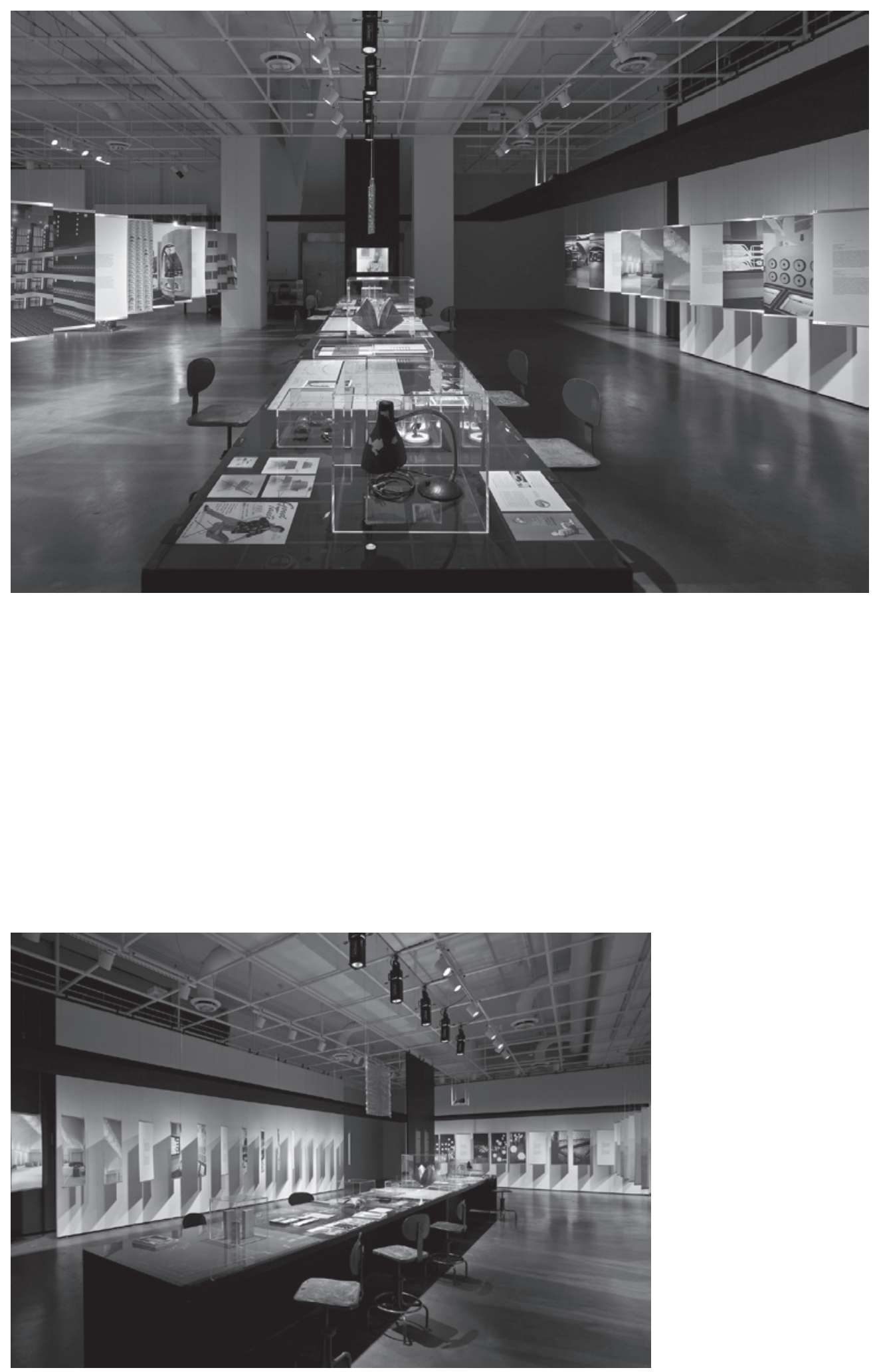

Vues de l'exposition

Photos: Michel Brunelle 
Le résultat est manifeste: chacune de ses interventions ou créations était pratiquement imbriquée, amalgamée, fusionnée avec l'environnement, témoignant sa compréhension des enjeux du projet architectural ou urbain sans pour autant nier son propre geste créateur. L'œuvre de Slater, exemple tangible et positif d'intégration de l'art à l'architecture, est le fruit d'une pratique qui s'est révélée être une pensée en mouvement, définitivement ancrée dans la recherche, mais surtout dans une volonté de maîtriser tant les matériaux que leur processus de transformation.

Pouvant être embrassée d'un seul coup d'œil, analogue aux jeux d'ombres des grillages métalliques de l'artiste, la mise en espace de l'exposition, réalisée par Georges Labrecque, était tracée sur le parcours didactique que formait la série de panneaux photographiques savamment suspendus, alternant la narration visuelle aux propos écrits. Cette muséographie, simultanément statique et animée, semblait reconstituer les feuillets du premier ouvrage à la mémoire de Slater. Le rythme de la présentation permettait une lecture aisée et aérée des textes, rappelant combien la simplicité d'un parcours de visite peut parfois être la solution la plus efficace à la diffusion d'un message. L'exposition visait dans un premier temps à circonscrire et à parler de la pratique du design et des «méthodes" du designer à la lumière des réalisations de Slater. Elle formulait aussi, dans un second temps, le mode opératoire qui anime l'École de design, celui de l'interdisciplinarité, fil d'Ariane de l'ouvrage accompagnant l'exposition². Ce concept d'interdisciplinarité qui traverse actuellement un grand nombre de pratiques est né notamment de ces expériences multidisciplinaires des années 1950, 1960 et 1970 qui tendaient vers des formes de synthèse de création. Les approches interdisciplinaires contemporaines se distinguent de ces expériences par leur composante critique qui interroge délibérément l'appareillage idéologique structurel des termes et des méthodes d'une pratique disciplinaire. Ces approches questionnent les processus dominants qui essaient de contenir la production créative. Ce faisant, elles génèrent de nouvelles formes de résistance, de nouveaux modes de connaissances et de compréhen$\operatorname{sion}^{3}$. Norman Slater est un exemple patent d'agent multidisciplinaire et l'exposition laissait clairement transparaître, par la variété des projets exposés, les fondements des pratiques plus actuelles du design qui cherchent à concrétiser la promesse des avant-gardes du début de siècle d'esthétiser chaque aspect de la vie sociale, y compris les objets et les comportements.

Le vernissage de l'exposition a été souligné par une mise en lumière dynamique (avec un des grillages de Slater comme motif), à l'extérieur du Centre de design. Cette primeur soulignait l'incorporation du Centre de design au «Parcours lumière » du Quartier des spectacles 

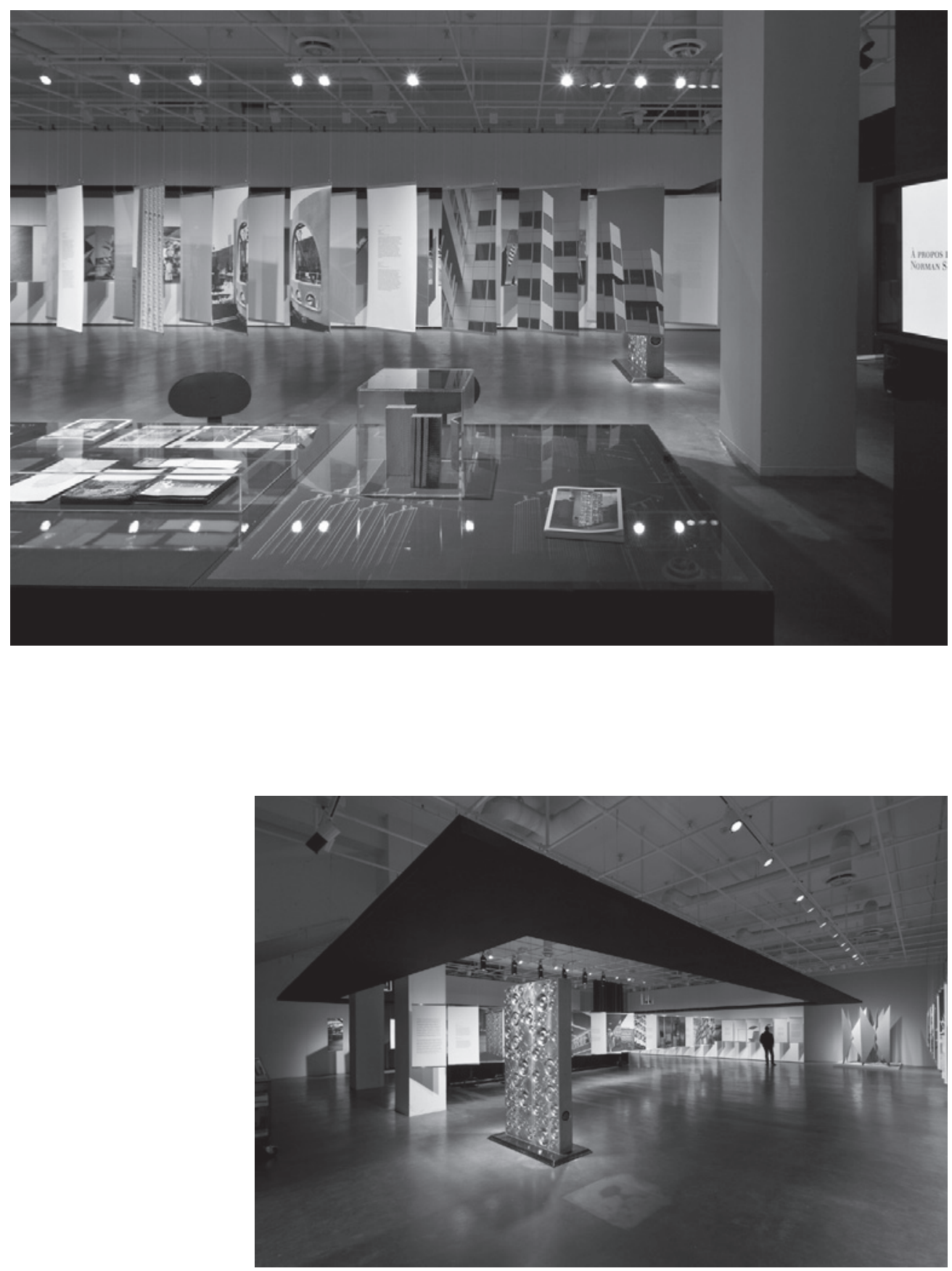

Vues de l'exposition

Photos: Michel Brunelle 
de Montréal ${ }^{4}$. Un circuit urbain venait prolonger l'espace d'exposition vers la ville: grâce à un dépliant-guide ${ }^{5}$, le visiteur pouvait replacer les œuvres de Slater dans leur contexte afin de mieux évaluer la contribution de ce dernier à la construction d'une l'image symbolique de la ville.

\section{Le Centre de design de l'UQAM}

Depuis plus de trente ans, la création en architecture, en design industriel, d'intérieur, de mode, urbain et graphique est exposée au Centre de design de I'UQAM. Unité informelle du Département de design qui avait été créé en 1975, initiative des professeurs France Vanlaethem, Alfred Halasa et Gérald Bochud, la Galerie de design de I'UOAM ${ }^{6}$ a ouvert ses portes en 1981. Si ses origines ont été modestes tant en termes d'espace ${ }^{7}$ que de financement, elle est rapidement devenue un point de rencontre obligé doté d'une forte image visuelle et une zone de diffusion démocratique pour étudiants et professeurs. Avec un programme culturel et pédagogique voué à la documentation de l'histoire du design, dont celle en train de se faire, à travers ses expositions et ses colloques, le Centre a offert - et offre toujours - ses espaces à la production locale et internationale. Dès les tout premiers débuts, les travaux étudiants étaient présentés en alternance avec ceux de créateurs plus illustres, dont des architectes tels que Mario Botta, Renzo Piano, Luigi Snozzi ou Tadao Ando; à des expositions thématiques devenues emblématiques, telles que Michel Dallaire, la beauté des formes utiles, Typomondo, $\operatorname{Re}(f)$ use ou comment l'art devient acceptable, Montreal 5; et à des expositions venues de grands musées, notamment le Centre Georges Pompidou. Lorsqu'en 1995 I'architecte Dan Hanganu crée le nouveau Pavillon de design (rue Sanguinet) en y intégrant le Centre de design de I'UQAM, c'est pour l'ouvrir à un public beaucoup plus grand. Depuis 1981, plus de 250 expositions $^{8}$ y ont été présentées, formant une collection éclectique, hétérogène, tous médias et supports confondus, afin de diffuser l'«art de concevoir», de communiquer et d'altérer le statu quo. Si la notoriété de l'École de design de l'UOAM n'est plus à faire au Canada et à l'étranger, c'est que le Centre de design a nécessairement contribué à la diffusion de sa production, celle d'un art où le medium devient message.

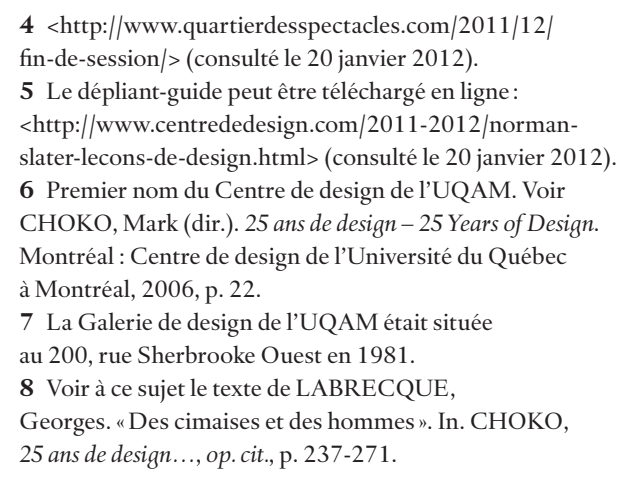

